\title{
The Influence of Professionalism to Job Satisfaction and Job Performance of the Physical Therapist
}

\author{
Jae Won Heo \\ Department of Physical Therapy, Graduate School, Sehan University, Yeongam-gun, Jeollanam-do, Korea
}

Purpose: This study was conducted in order to understand the influence of professionalism on job satisfaction and job performance of the physical therapist.

Methods: Subjects were 148 physical therapists. We conducted a questionnaire survey on professionalism, job satisfaction, and job performance. Professionalism questions were 'Compliance of professional organization', 'Beliefs about public service', 'Autonomy', 'Beliefs about self-regulation', and 'Vocation awareness of the profession'. A regression analysis was then performed.

Results: The subjects' average score for professionalism was $3.18 \pm 0.31$ points. The lowest score for satisfaction was observed for 'Compliance of professional organization.' Professionalism had an effect on job satisfaction. 'Vocation awareness of the profession $(\beta=0.44$, $p<0.001)$ ', 'Beliefs about public service $(\beta=0.25, p<0.01)$ ', and 'Autonomy $(\beta=0.17, p<0.05)$ ' had an effect on job satisfaction. $R$ square $=0.46$ (adjusted $R$ square $=0.45$ ). Professionalism had an effect on job performance. 'Autonomy $(\beta=0.29), p<0.001$ )', 'Vocation awareness of the profession $(\beta=0.22, p<0.05)$ ', and 'Beliefs about self-regulation $(\beta=0.18, p<0.05)$ ' had an effect on job performance. $R$ square $=0.25$ (adjusted $R$ square $=0.22$ ).

Conclusion: The results of this study indicate that in order to improve job satisfaction and job performance, it is necessary to improve professionalism. Therefore physical therapists should improve their professionalism ('Compliance of professional organization', 'Beliefs about public senvice', 'Autonomy', 'Beliefs about self-regulation', and 'Vocation awareness of the profession') in order to improve their job satisfaction and job performance. In addition, research to improve professionalism of the physical therapist should be continued.

Keywords: Professionalism, Job satisfaction, Job performance

\section{서 론}

현대인은 건강에 대한 관심과 그에 대한 욕구가 증가되고 있으며 이 에 따라 건강 전문직에 대한 사회적 기대와 수요가 늘어나고 있는 추 세이다. 전문 직업은 특수한 지식과 기술을 토대로 광범위한 기능적 자율성을 갖고 수행된 과업의 성과에 따라 보수를 받는 직업을 말하 는데 물리치료사는 이러한 대표적인 건강 전문 직업에 속한다.1

Hall은 전문 직업성의 공통 속성으로 구조적 속성과 태도적 속성 을 제시하고 있다. 구조적 속성으로 종일제 직업(full time occupation), 지식기반의 전문적인 교육 훈련기관의 존재, 전문가 연합회, 내외부 적으로 기대되는 윤리규범의 존재를 제시하고 있으며, 태도적 속성 은 전문가 집단의 준거성, 공공봉사에의 신념, 자기규제, 해당분야에 대한 소명의식, 전문가적 자율성이 해당된다.
2014년 보건복지통계연보에 따른 2013년 기준 우리나라 물리치료 사 현황은 남자 16,562 명, 여자 31,148 명으로 전체 47,710 명의 물리치료 사가 등록되어 있는 것으로 나타났다. 이는 2003년 21,153명에 비해 2 배 이상 증가된 수이며, 등록된 전체 의료기사의 약 $16.9 \%$ 에 해당하 는 수치로 물리치료사가 특수성과 전문성을 추구하며 전문 직업으 로서 자리잡고 있음을 보여준다. ${ }^{3}$ 그러나 대부분의 물리치료사들은 병원, 재활병원, 요양병원, 의원, 보건소 등에서 독립적인 운영 및 지도 를 할 수 없는 근무환경에 놓여있으며, 이러한 근무환경은 전문 직업 적 정체성에 혼란을 야기시킬 수 있다. 전문 직업적 정체성은 자신이 소속되어 있는 집단을 자신과 같이 특정하거나 같은 범주로 동일 시 하는 것으로 전문 직업적 정체성의 혼란은 직무에 대한 만족과 몰입 도를 저하시키게 되며 결과적으로 조직의 효율성을 떨어지게 한다. ${ }^{4}$

직무만족이란 각 개인이 자기 직무와 관련하여 경험하는 모든 정
Received Jul 23, 2015 Revised Aug 20, 2015

Accepted Aug 21, 2015

Corresponding author Jae Won Heo

E-mail1831317@hanmail.net
Copylight (C2015 The Korea Society of Physical Therapy

This is an Open Access article distribute under the terms of the Creative Commons Attribution Non-commercial License (Http:// creativecommons.org/license/by-nc/3.o.) which permits unrestricted non-commercial use, distribution, and reproduction in any medium, provided the original work is properly cited. 
서의 총화 또는 이러한 정서의 균형 상태에서 기인되며, 임금이나 관 리, 직무내용 등 직무조건에 대한 정서적 반응으로서 보통 사기나 직

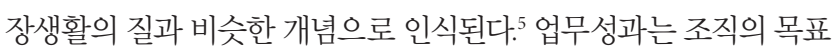
달성을 위한 과업의 실행 정도로써 조직 구성원이 지니는 노력, 능력, 역할 지각에 의한 달성 여부를 가리키는 조직 행동의 일면으로 정의 될 수 있다. 직무만족과 업무성과 간의 관계에 관한 선행연구들을 살 펴보면 $\mathrm{Ban}^{7}$ 은 간호사들을 대상으로 한 연구에서 직무만족도가 높 을수록 간호업무성과가 높게 나타난다고 보고하였다. 간호조무사를 대상으로 한 연구에서도 직업만족과 전문직업성이 조직몰입에 정의 효과를 나타낸다고 하였다. 그 밖에 다양한 직업에서 직무만족과 업 무성과 간에 관련성을 밝힌 연구들이 국내외에서 이루어졌다.

국내에서 이루어진 물리치료사의 전문직업성, 직무만족, 물리치료 사의 업무성과 등에 관한 연구들을 살펴보면 Cheon'은 물리치료사 의 일반적 특성과 근무실태를 조사하고 이에 따른 직무만족도간의 관계를 조사하였다. Ahn 등 ${ }^{10}$ 역시 물리치료사의 일반적인 특성과 근 무실태를 바탕으로 직무만족과 직장애착의 정도를 연구한바 있다.

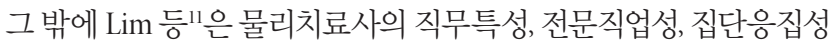
을 통해 물리치료사의 조직시민행동에 관한 연구 모형을 설계하였 다. 또한 Lee 등12은 전남 동부권 지역 내 물리치료사 129 명을 대상으 로 물리치료사의 근무환경과 직무만족도에 관한 연구를 통해 업무 환경과 업무량에 따른 직무만족도를 살펴본 바 있다.

이처럼 물리치료사의 직무만족과 업무성과에 관련된 국내 연구 들이 지속적으로 이어지고 있으나 다른 전문 직업이나 병원 종사자 들에 관한 연구들에 비해 다양한 접근이 이루어지고 있지 못한 실정 이다. 또한 물리치료사의 전문직업성이 그들의 직무만족이나 업무성 과에 어떠한 영향을 미치는지에 대한 연구들은 더욱 제한적이다. 따 라서 본 연구에서는 물리치료사들의 전문직업성을 분석하고 이러한 전문직업성이 직무만족도와 물리치료 업무성과에 어떠한 영향을 미 치는지 알아보고자 한다. 이를 통해 전문직업으로서 물리치료사의 중요성에 대해 확인하고 물리치료사의 업무능력 향상을 위해 필요한 기초자료를 제공하고자 한다.

\section{연구방법}

\section{1. 연구대상}

본 연구는 광주. 전남 지역의 일부 종합병원, 재활병원, 요양병원, 의 원 등에서 근무하고 있는 물리치료사를 대상으로 이루어졌다. 대상 자는 지역 내 의료기관에 근무 중인 물리치료사들을 대상으로 무작 위 선정하였으며, 설문에 앞서 본 연구의 목적에 대해 충분히 설명하 고 설문에 참여할 의사를 밝힌 자로 하였다. 설문방식은 물리치료사 가 근무하는 기관에 직접 방문하여 배포 후 회수하는 것을 기본으로
하였으며, 부득이한 경우 우편 혹은 전자우편을 통해 진행하였다. 전 체 178 부의 설문지 중 설문내용에 누락된 부분이 있는 설문지를 제외 한 148 부를 분석하였다.

\section{2. 연구방법}

1) 측정지표

(1) 전문직업성

전문직업성이란 클라이언트에게 서비스를 제공하는 데 있어 자신이 활용할 수 있는 전문직에 대한 지식, 기술 및 자격과 그 가치와 윤리 를 고수하는 태도와 행동을 말한다. ${ }^{3}$

본 연구에서 사용한 전문직업성 측정도구는 Hallo이 개발한 Professionalism Inventory Scale을 기반으로 하여 Snizek ${ }^{14}$, Baek과 Soo ${ }^{15}$ 가 수 정한 내용을 사용하여 측정하였다.

설문 내용은 준거성 5 문항, 공공서비스에 대한 신념 5 문항, 자율성 5 문항, 자체규제에 대한 신념 5 문항, 소명의식 5 문항 등 총 25 문항으 로 구성되어있으며, Likert 5점 척도 5점 '매우 그렇다', 1점 '전혀 그렇지 않다로 부여하여 측정하였다. 점수가 높을수록 전문직업성이 높은 것을 의미한다. 본 도구의 신뢰도는 Baek과 $S_{0 o}{ }^{15}$ 의 연구에서는 Cronbach's $\alpha=0.82$ 였으며, 본 연구에서는 Cronbach's $\alpha=0.80$ 이었다.

(2) 직무만족

직무만족은 직무와 직무조건과 관련하여 형성되는 것으로서 자신의 직무에 대한 평가 혹은 직무경험을 통해 느끼게 되는 즐거운 혹은 긍 정적인 감정의 상태로 정의된다. ${ }^{16}$

본 연구에서 사용한 직무만족 측정도구는 Flippo와 Seberhagen의 직무만족 요인과 물리치료사의 직무만족에 관한 선행연구들에서 사 용한 것을 참조하여 연구 목적에 맞게 변형하여 사용하였다.10, 12, 17

직무만족을 측정하기 위한 설문은 직무에 관한 사항 6 문항, 감독 에 관한 사항 8 문항, 보수에 관한 사항 5 문항, 승진에 관한 사항 5 문항, 동료에 관한 사항 5 문항, 기관에 관한 사항 5 문항, 이직에 관한 사항 4 문항으로 구성하였다. 측정은 Likert 5점 척도로써 5점 '매우 그렇다', 1 점 '전혀 그렇지 않다' 로 부여하여 점수가 높을수록 직무만족이 높 음을 의미한다. 본 연구에서 신뢰도는 Cronbach's $\alpha=0.92$ 였다.

\section{(3) 업무성과}

일반적으로 업무성과는 사회적, 생물학적 그리고 기계적 체계에도 적용될 수 있는 개념으로서 특정의 기대, 목적 혹은 목표의 달성 정 도를 의미한다. ${ }^{18}$

물리치료 업무성과 측정도구는 Park $^{17}$ 과 Kim${ }^{19}$ 이 구성한 업무수행 평가도구를 물리치료사에게 맞게 수정하여 사용하였다.

문항은 독자적 물리치료활동 9문항, 비독자적 물리치료활동 9 문 
항, 대인관계 활동 5 문항으로 총 23 문항이며, Likert 5점 척도로 5점 '매우 그렇다', 1점 '전혀 그렇지 않다' 로 총점 23점에서 115점으로 점 수가 높을수록 물리치료 업무성과 정도가 높음을 의미한다. 본 도구 의 신뢰도는 Park ${ }^{17}$ 의 연구에서는 Cronbach's $\alpha=0.96$ 이었으며, 본 연 구에서는 Cronbach's $\alpha=0.95$ 였다.

\section{2) 자료분석}

본 연구를 위해 수집된 물리치료사들의 설문자료는 SPSS 18.0 프로그 램을 사용하여 분석하였다. 대상자들의 일반적 특성과 전문직업성 을 알아보기 위해 빈도분석, 평균, 표준편차를 이용하였고, 물리치료 사의 전문직업성이 직무만족도와 업무성과에 미치는 영향을 알아보 기 위해 단계적 다중회귀분석을 실시하였다. 통계적 유의수준 $\mathrm{p}<$ 0.05 로 정하였다.

\section{결 과}

\section{1. 대상자의 일반적 특성}

전체 대상자의 $50.7 \%$ 가 4 년제 이상 대학졸업자로 나타났으며, 총 근 무경력은 $85.8 \%$ 가 5 년 미만인 것으로 나타났다. 월 보수는 대부분이 250 만원 이하로 나타났으며, $5.4 \%$ 만이 그 이상의 보수를 받고 있는 반

Table 1. General characteristics

\begin{tabular}{|c|c|c|}
\hline Classification & Division & $\begin{array}{l}\text { Distribution } \\
(\mathrm{N}=148)(\%)\end{array}$ \\
\hline \multirow[t]{2}{*}{ Gender } & Male & $74(50.0)$ \\
\hline & Female & $74(50.0)$ \\
\hline \multirow[t]{5}{*}{ Age (year) } & $21-25$ & $40(27.0)$ \\
\hline & $26-30$ & $64(43.2)$ \\
\hline & $31-35$ & $19(12.8)$ \\
\hline & $36-40$ & $20(13.5)$ \\
\hline & $41<$ & $5(3.4)$ \\
\hline \multirow[t]{2}{*}{ Marital status } & Married & $56(37.8)$ \\
\hline & Unmarried & $92(62.2)$ \\
\hline \multirow[t]{4}{*}{ Level of education } & College (3-year) & $73(49.3)$ \\
\hline & University (4-year) & $48(32.4)$ \\
\hline & Graduate student & $15(10.1)$ \\
\hline & Finish graduate school & $12(8.1)$ \\
\hline \multirow[t]{5}{*}{ Total career (year) } & $<1$ & $34(23.0)$ \\
\hline & $1-3$ & $25(16.9)$ \\
\hline & $3-5$ & $35(23.6)$ \\
\hline & $5-10$ & $32(21.6)$ \\
\hline & $10<$ & $22(14.9)$ \\
\hline \multirow[t]{5}{*}{ Monthly salary (10,000 won) } & $100-150$ & $49(33.1)$ \\
\hline & $151-200$ & $55(37.2)$ \\
\hline & $201-250$ & $36(24.3)$ \\
\hline & $251-300$ & $1(0.7)$ \\
\hline & $301<$ & $7(4.7)$ \\
\hline
\end{tabular}

면 150 만원 이하의 보수를 받는 대상자는 $33.1 \%$ 를 차지했다(Table 1).

\section{2. 물리치료사의 전문직업성}

대상자들의 전문직업성에 대해 살펴본 결과 전체 평균 $3.18 \pm 0.31$ 점으 로 나타났으며, 전문조직의 준거성 항목에서 평균 $2.75 \pm 0.53$ 점으로 '보통이다(3점)'보다 낮은 점수를 보여 가장 낮게 나타났다. 나머지 공 공서비스에 대한 신념(3.33 \pm 0.44$)$, 자율성(3.16 \pm 0.46$)$, 자체규제에 대 한 신념(3.27 \pm 0.48$)$, 직업에 대한 소명의식(3.41 \pm 0.62$)$ 항목에서는 보 통 이상의 점수를 보였다.

\section{3. 전문직업성이 직무만족에 미치는 영향}

전문직업성이 물리치료사의 직무만족에 미치는 영향을 알아보기 위 해 단계적 다중회귀분석을 시행하였다. 직무만족을 설명하기 위한 독립변수로 전문직업성을 평가하는 전문조직의 준거성, 공공서비스 에 대한 신념, 자율성, 자체규제에 대한 신념, 직업에 대한 소명의식을 투입한 결과 F 통계값 $24.55(\mathrm{p}<0.001)$ 로 직무만족에 대해 유의하게 설명하고 있다. 이때 독립변수는 종속변수를 약 $46.4 \%$ 설명한다.

개별 독립변수의 종속변수에 대한 기여도와 통계적 유의성을 검 정한 결과 유의수준 0.05 에서 직무만족에 유의하게 영향을 미치는 독립변수는 공공서비스에 대한 신념 $(\mathrm{t}=3.47, \mathrm{p}=0.001)$, 자율성 $(\mathrm{t}=2.62$, $\mathrm{p}=0.010)$, 직업에 대한 소명의식 $(\mathrm{t}=6.03, \mathrm{p}<0.001)$ 이며, 독립변수의 상 대적 기여도를 나타내는 표준화 계수에 의하면 직업에 대한 소명의 식, 공공서비스에 대한 신념, 자율성 순으로 직무만족에 영향을 미치 고 있다(Table 2).

\section{4. 전문직업성이 업무성과에 미치는 영향}

전문직업성이 물리치료사의 업무성과에 미치는 영향을 알아보기 위 해 직무만족에 대한 분석방법과 동일하게 시행하였다. 전문조직의

Table 2. Professionalism that influences in the job satisfaction

\begin{tabular}{|c|c|c|c|c|c|c|}
\hline & & B & $\beta$ & $t$ & $p$ & VIF \\
\hline \multirow{6}{*}{$\begin{array}{l}\text { Job } \\
\text { satisfaction }\end{array}$} & Constant & 18.07 & & & & \\
\hline & $\begin{array}{l}\text { Compliance } \\
\text { of professional } \\
\text { organization }\end{array}$ & 0.37 & 0.02 & 0.38 & 0.701 & 1.03 \\
\hline & $\begin{array}{l}\text { Beliefs about } \\
\text { public service }\end{array}$ & 9.20 & 0.25 & 3.47 & $0.001 * *$ & 1.32 \\
\hline & Autonomy & 5.98 & 0.17 & 2.62 & $0.010^{*}$ & 1.05 \\
\hline & $\begin{array}{l}\text { Beliefs about } \\
\text { self-regulation }\end{array}$ & 4.10 & 0.12 & 1.84 & 0.068 & 1.08 \\
\hline & $\begin{array}{l}\text { Vocation } \\
\text { awareness of } \\
\text { the profession }\end{array}$ & 11.69 & 0.44 & 6.03 & $<0.001^{* * *}$ & 1.38 \\
\hline
\end{tabular}

R square $=0.46$, adjusted $R$ square $=0.45, F=24.55(p<0.001)$.

${ }^{*} p<0.05 ;{ }^{* *} p<0.01 ;{ }^{* * *} p<0.001$. 
Table 3. Professionalism that influences in the job performance

\begin{tabular}{llccccc}
\hline & & $\mathrm{B}$ & $\beta$ & $\mathrm{t}$ & $\mathrm{p}$ & $\mathrm{VIF}$ \\
\hline Job & $\begin{array}{l}\text { Constant } \\
\text { satisfaction }\end{array}$ & 34.67 & & & & \\
$\begin{array}{c}\text { Compliance } \\
\text { of professional } \\
\text { organization }\end{array}$ & -0.84 & -0.09 & -1.18 & 0.241 & 1.03 \\
$\begin{array}{c}\text { Beliefs about } \\
\text { public service } \\
\text { Autonomy }\end{array}$ & 1.79 & 0.08 & 0.91 & 0.367 & 1.32 \\
$\begin{array}{c}\text { Beliefs about } \\
\text { self-regulation } \\
\text { Vocation } \\
\text { awareness of } \\
\text { the profession }\end{array}$ & 3.94 & 0.79 & 0.18 & 2.37 & $0.019^{*}$ & 1.08 \\
& & 0.22 & 2.61 & $0.010^{*}$ & 1.38 \\
\hline
\end{tabular}

R square $=0.25$, adjusted $R$ square $=0.22, F=9.34(p<0.001)$.

${ }^{*} p<0.05 ;{ }^{* *} p<0.001$.

준거성, 공공서비스에 대한 신념, 자율성, 자체규제에 대한 신념, 직업 에 대한 소명의식을 투입한 결과 F 통계값 $9.34(\mathrm{p}<0.001)$ 로 업무성과 에 대해 유의하게 설명하고 있다. 이때 독립변수는 종속변수를 약 $24.8 \%$ 설명하고 있다.

개별 독립변수의 종속변수에 대한 기여도와 통계적 유의성을 검 정한 결과 유의수준 0.05 에서 업무성과에 유의하게 영향을 미치는 독립변수는 자율성 $(\mathrm{t}=3.82, \mathrm{p}<0.001)$, 자체규제에 대한 신념 $(\mathrm{t}=2.37$, $\mathrm{p}=0.019)$, 직업에 대한 소명의식 $(\mathrm{t}=2.61, \mathrm{p}=0.010)$ 이며, 독립변수의 상 대적 기여도를 나타내는 표준화 계수에 의하면 자율성, 직업에 대한 소명의식, 자체규제에 대한 신념 순으로 업무성과에 영향을 미치는 것으로 나타났다(Table 3).

\section{고 찰}

본 연구는 광주·전남 직역의 일부 물리치료사들을 대상으로 전문직 업성을 분석하고, 전문직업성이 물리치료사의 직무만족과 업무성과 에 미치는 영향을 알아보기 위해 실시되었다.

첫 번째, 본 연구에서 물리치료사의 전문직업성은 '전문조직의 준 거성', 공공서비스에 대한 신념', '자율성, '자체규제에 대한 신념', '직업 에 대한 소명의식 항목들에 대한 전체 평균에서 보통 이상으로 나타 났다. 이러한 결과를 다른 직종과 비교해보면 치과위생사의 경우 143 명을 대상으로 한 연구에서 Likert 5점 척도 기준으로 3.04점, 사회복 지사의 경우 172 명을 대상으로 한 연구에서 3.54 점, 간호사의 경우 169 명의 수술실 임상간호사를 대상으로 한 연구에서 3.18점 등으로 본 연구 결과와 유사한 것으로 나타났다. ${ }^{2022}$ 이를 통해 물리치료사의 경우 주관적 평가 시 다른 직종과 유사한 보통 이상의 전문직업성을 갖추고 있음을 확인 할 수 있었다.

두 번째, 물리치료사의 전문직업성이 직무만족에 미치는 영향을
살펴본 결과 직무만족에 대한 전문직업성의 설명력은 약 $46.4 \%$ 였으 며, 항목별로는 소명의식, 공공서비스에 대한 신념, 자율성 순서의 기 여도를 나타냈다.

다른 직종에서 전문직업성과 직무만족의 관계에 대한 선행연구들 을 살펴보면 임상간호사 329 명을 대상으로 한 연구에서 전문직업성 과 직무만족이 정의 상관관계가 있다고 밝혔으며 하위영역 중 자율 성, 자체규제에 대한 신념, 직업에 대한 소명의식이 직무만족과 정의 상관관계를 보인다고 하였다. 또한 전문직업성이 직무만족에 미치는 희귀모형에서 $10 \%$ 의 설명력을 나타냈고 하위영역 중 자율성, 직업에 대한 소명의식이 직무만족에 영향을 미치는 것으로 나타났다.23 임상 간호사를 대상으로 한 다른 연구에서도 전문직업성과 직무만족간 상관관계를 분석한 결과 전문직업성의 총점과 직무만족 총점 간의 유의한 정의 상관관계를 보였고 일부 하위영역들 간에도 정의 상관 관계가 있음을 밝혔다. ${ }^{24}$ 사회복지사의 전문직업의식과 직무만족에 영향을 미치는 요인에 관한 연구에서 역시 전문직업의식이 직무만족 에 유의한 영향을 주는 요인으로 나타났다.21

물리치료사를 대상으로 한 선행연구들을 살펴보면 운동처방사와 물리치료사 596명을 대상으로 한 연구 결과 전문직업적 정체성 항목 중 소명의식, 자율성에 대한 신념, 전문조직의 활용, 서비스에 대한 신 념 항목 순으로 직무만족에 영향을 미치며 설명력은 $36.4 \%$ 인 것으로 나타났다..$^{25}$ 또한 221 명의 물리치료사를 대상으로 자신이 속한 전문 직에 종사하는 직업인으로서 자기 자신에 대한 정신적 지각에 해당 하는 전문직 자아개념과 직무만족과의 관계연구에서 두 변수 간에 정의 상관관계가 있음을 밝혔다. ${ }^{26}$

이러한 결과들을 볼 때 물리치료사 역시 회귀식의 설명력과 전문 직업성의 일부 하위영역들에서 차이는 나타나지만 다른 직종과 마찬 가지로 자신의 직업에 대한 주관적인 전문직업성이 직무만족도에 영 향을 미치는 것을 확인할 수 있다. 그러나 전문직업성을 평가하는 척 도가 다른 외부의 평가가 아닌 주관적이고 내적인 태도요소에 초점 을 두고 있다는 점은 고려되어야 할 것으로 사료된다. 따라서 전문직 업성에 대한 보다 객관적인 평가를 위한 연구들이 이루어져야 할 필 요가 있을 것으로 생각된다.

마지막으로 전문직업성이 물리치료 업무성과에 미치는 영향을 살 펴본 결과 회귀모형에서 설명력은 약 $24.8 \%$ 였으며, 자율성, 직업에 대 한 소명의식, 자체규제에 대한 신념 순서로 업무성과에 영향을 미치 는 것으로 나타났다.

우선 전문직업성과 업무성과 간의 관계에 관한 간호분야의 연구 들을 살펴보면 임상간호사의 전문직 태도와 간호수행 정도에 관한 연구에서 전문직 태도의 항목으로 전문직 교육수준, 사회이익추구, 전문직 단체 조직, 책임감, 실제성, 자율성, 윤리성 등이 포함되었고 이 러한 요소들이 간호수행과 정의 상관관계 $(\mathrm{r}=0.717, \mathrm{p}<0.001)$ 를 나타 
내는 것으로 보고하였다. ${ }^{27}$ 또한 수술실 간호사를 대상으로 한 연구 에서 역시 전문직업성과 업무수행능력 간에 유의한 상관관계 $(\mathrm{r}=0.316, \mathrm{p}<0.001)$ 가 있는 것으로 나타났다. 이 연구에서 사용된 전 문직업성 평가는 전문조직의 준거성, 공공서비스에 대한 신념, 자율 성, 자체 규제에 대한 신념, 직업에 대한 소명의식 등을 포함하고 있어 본 연구에서 사용된 항목들과 유사하다.22 마찬가지로 본 연구와 유 사한 전문직업성 평가 항목을 사용한 $\operatorname{Shin}^{28}$ 은 임상간호사의 전문직 업성과 조직효과성의 관계연구에서 전문직업성이 간호업무수행과 정의 상관관계 $(r=0.482, p<0.001)$ 가 있다고 보고하였으며, 전문직업 성의 세부 항목에서도 전문조직의 준거성 $(\mathrm{r}=0.161 \mathrm{p}=0.036)$, 공적 서 비스에 대한 신념 $(r=0.177, \mathrm{p}=0.021)$, 자율성 $(\mathrm{r}=0.317, \mathrm{p}<0.001)$, 자체규 제에 대한 신념 $(\mathrm{r}=0.378, \mathrm{p}<0.001)$, 직업에 대한 소명의식 $(0.387$, $\mathrm{p}<0.001)$ 등과 전체적인 간호업무수행 정도와 상관관계가 있다고 밝 혔다.

물리치료사의 전문직업성이 업무성과에 미치는 영향에 관한 연구 들이 제한적이므로 그와 유사한 연구들을 살펴보면 Park과 $\mathrm{Heo}^{29}$ 는 물리치료사의 전문직업성, 직무만족, 업무성과 간의 상관관계 연구 에서 전문직업성과 업무성과 간에 약한 양의 선형관계 $(\mathrm{r}=0.238$, $\mathrm{p}=0.004)$ 를 보인다고 밝힌 바 있다. 또한 Lee ${ }^{25}$ 는 운동처방사와 물리 치료사를 대상으로 직업정체성으로 전문조직 활용, 서비스에 대한 신념, 소명의식, 자율성 등의 항목을 사용하여 조직몰입에 미치는 영 향을 연구한 결과 회귀식의 설명력 $40.4 \%$ 로 직업정체성이 조직몰입 에 영향을 미치는 것으로 나타났다. 조직몰입에 영향을 미치는 요인 으로는 소명의식과 자율성 순서였다. 비록 조직몰입이 본 연구에서 살펴본 업무성과를 직접적으로 설명하지는 못하지만 조직 몰입도가 높을수록 성과가 높아질 가능성이 높고 조직몰입이 조직효과성의 유용한 지표가 된다. ${ }^{30}$

이러한 결과들을 살펴볼 때 전문직업성이 업무를 수행하는 데 영 향을 미친다는 선행연구들을 뒷받침한다. ${ }^{31}$ 비록 물리치료사를 대상 으로 한 전문직업성의 영향에 대한 연구들이 제한적인 이유로 다른 전문직업들과의 간접적인 비교를 실시하였으나 물리치료사 역시 다 른 전문직업들과 마찬가지로 전문직업성의 향상을 통해 물리치료 업 무성과를 향상시킬 수 있을 것으로 예상할 수 있다. 특히 본 연구의 결과에서 나타났듯이 물리치료사의 자율성을 보장하고 물리치료사 스스로 자체규제에 대한 신념과 소명의식을 함양한다면 물리치료 업무영역이 보다 전문성을 가질 수 있을 것으로 기대되며, 이를 통해 보다 높은 물리치료 업무성과를 달성할 수 있을 것으로 사료된다.

본 연구에서는 대상자 선정에서 일부 지역으로 제한되어 다양한 지역의 물리치료사들에 대한 분석이 이루어지지 못한 점을 이유로 전체 물리치료사로 일반화하는 데 어려움이 있을 수 있을 것으로 생 각된다. 그러나 전남 동부권 지역 물리치료사 120 명의 근무환경과 직
무만족도 조사연구와 서울시 공공의료기관 물리치료사 57 명을 대상 으로 한 직무만족도 연구 등을 통해 볼 때 본 연구의 대상자를 통해 서도 의미있는 분석이 될 수 있을 것으로 사료된다.12,32 따라서 본 연 구에서 물리치료사의 전문직업성에 관한 세부 항목들을 살펴보고 이를 통해 물리치료사의 직무만족도와 업무성과에 영향을 미칠 수 있는 요인들을 구체적으로 확인할 수 있었다는 점에서 의의가 있을 것으로 생각된다. 이러한 결과들은 향후 전문직업인으로서 물리치료 사의 업무환경과 처우개선 등에 필요한 근거자료로 활용될 수 있을 것으로 사료되며, 물리치료사 스스로 전문직업인으로서의 자각과 업무성과 향상을 위해 노력해야 할 부분들에 대한 객관적 자료를 제 시할 수 있을 것으로 기대된다. 또한 물리치료사의 전문직업성 함양 과 업무성과 향상을 위해 보다 많은 객관화된 평가와 연구들이 이루 어져야 할 것으로 생각된다.

\section{REFERENCES}

1. Kown HC, Chung YI. An analysis on demand and supply for medical rehabilitation professionals in Korea. J Kor Phys Ther. 1997;9(1):19-35.

2. Hall RH. Professionalism and bureaucratization. Am Sociol Rev. 1968;63:92-104.

3. Ministry of health and welfare statistical year book. Ministry of Health \& Welfare. 2014.

4. Choi DH. A Study on job satisfaction of physical therapy. Dnakook University. Dissertation of Master's Degree. 1992.

5. Park WY. A Study on the channels through which the job satisfaction affects the job commitment and the organizational commitment. Kyunghee University. Dissertation of Master's Degree. 2002.

6. Kim SH, Lee YM. Effects of job satisfaction and organizational commitment on performance of part-time employees. Journal of Human Resource Management Research. 2013;20(1):89-105.

7. Ban YR. The effect of communication satisfaction and job satisfaction on the nursing performance of hospital nurses. Chungang University. Dissertation of Master's Degree. 2002.

8. Kim SI. An effect of the job satisfaction and professional expertise recognition of nurse aides upon organizational commitment. Inje University. Dissertation of Master's Degree. 2011.

9. Cheon JK. A study on the service performance and job satisfaction of physical therapists. J Kor Phys Ther. 1991;3(1):9-38.

10. Ahn SY, Kim WJ, Huh YB. Working conditions, job satisfaction and organizational commitment of physical therapists. J Kor Phys Ther. 2002;14(4):308-22.

11. Lim JD, Lee KH, Kim WJ. Influence of individual and job characteristics, professional job perception, and group cohesiveness on organizational citizenship behavior of physical therapists in hospitals. KSHM. 2003; 8(2):70-92.

12. Lee HS, Song HK, Kim HN et al. The study work conditions and job satisfaction of physical therapist in Jeonnam east area. J Kor Phys Ther. 2005;17(1):80-95.

13. Barker RL. The Social Work Dictionary. Silver Spring, Maryland, 
NASW, 2003.

14. Snizek WE. Hall's professionalism scale: An empirical reassessment. American Sociological Review. 1972;3:109-14.

15. Baek HC, Soo YU. Translation and validation of Korean version of Hall's professionalism inventory. J Korean Acad Nurs Admin. 2007;13(4):50915

16. Locke EA. The nature and cause of job satisfaction : Hand book of industrial and organizational psychology. Chicago, Rand McNally, 1976.

17. Park SA. An exploratory study on the relationship between leadership style and performance in Korean nursing units. The Seoul Journal of Nursing. 1990;4(1):45-61.

18. Stewart J, Walsh K. Performance measurement: When performance can never be finally defined. Public Mcney \& Management. 1994;14(2):45-9.

19. Kim WB. A study on the self-leadership and job satisfaction of workers in the companies. Younsei University. Dissertation of Master's Degree. 2001.

20. Yeon MR. The relationship of professionalism and organizational commitment with intention to change jobs among dental hygienists. Ajou University. Dissertation of Master's Degree. 2011.

21. Lee SE. Factors affecting the professionalism and job satisfaction of Daejeon social worker. Daejeon University. Dissertation of Master's Degree. 2007.

22. Hwang SM. Competency performance ability, professionalism and job satisfaction in operating room nurses. Kongju National University. Dissertation of Master's Degree. 2012.

23. Kwon KJ, Chu MS, Kim JA. The impact of nursing professionalism on the nursing performance, job satisfaction and retention intention among clinical nurses. J Korean Acad Nurs Admin. 2009;15(2):182-92.

24. Cha IJ. Clinical nurses' professionalism and job satisfaction, organization commitment. Hanyang University. Dissertation of Master's Degree. 2008.

25. Lee SG. Effect of identity that exercise and physical therapist perceives on organization commitment, job commitment and job satisfaction. Kyunghee University. Dissertation of Doctorate Degree. 2009.

26. Lee KH, Jung BO. A Study on the relationship between professional selfconcept and job satisfaction of physical therapists. J Kor Phys Ther. 2009; 21(3):109-18.

27. Choi SH. A Study on nursing professional attitude and nursing performance of clinical nurses. Ewha Womans University. Dissertation of Master's Degree. 1997.

28. Shin HS. A study on the relationship between professionalism and organizational effectiveness of clinic nurses'. Hanyang University. Dissertation of Master's Degree. 2009.

29. Park SK, Heo JW. Correlation between professionalism, job satisfaction and job performance of the physical therapist. J Kor Phys Ther. 2015; 27(1):12-7.

30. Steers RM. Antecedents and outcomes of organizational commitment. Adm Sci Q. 1977;22:46-56.

31. Boyt TE, Lusch RF, Naylor G. The role of professionalism in determining job satisfaction in professional services: A study of marking researchers. J Serv Res. 2001;3(4):321-30.

32. Moon JK, Song BK, Hwang BY. A Study on job satisfaction among physical therapists in the public health centers of the Seoul metropolitan area. J Kor Phys Ther. 2010;22(2):61-8. 